\title{
MRI of intact plants
}

\author{
Henk Van As · Tom Scheenen · Frank J. Vergeldt
}

Received: 3 July 2009/ Accepted: 29 July 2009/Published online: 27 August 2009

(c) The Author(s) 2009. This article is published with open access at Springerlink.com

\begin{abstract}
Nuclear magnetic resonance imaging (MRI) is a non-destructive and non-invasive technique that can be used to acquire two- or even three-dimensional images of intact plants. The information within the images can be manipulated and used to study the dynamics of plant water relations and water transport in the stem, e.g., as a function of environmental (stress) conditions. Non-spatially resolved portable NMR is becoming available to study leaf water content and distribution of water in different (sub-cellular) compartments. These parameters directly relate to stomatal water conductance, $\mathrm{CO}_{2}$ uptake, and photosynthesis. MRI applied on plants is not a straight forward extension of the methods discussed for (bio)medical MRI. This educational review explains the basic physical principles of plant MRI, with a focus on the spatial resolution, factors that determine the spatial resolution, and its unique information for applications in plant water relations that directly relate to plant photosynthetic activity.
\end{abstract}

Keywords Xylem $\cdot$ Phloem $\cdot$ Flow conducting area Hydraulic conductance - Water content - Storage pools . Dynamic behavior

H. Van As $(\square)$. T. Scheenen · F. J. Vergeldt

Laboratory of Biophysics and Wageningen NMR Centre,

Wageningen University, Dreijenlaan 3, 6703 HA Wageningen,

The Netherlands

e-mail: Henk.vanas@wur.nl

URL: www.bip.wur.nl and www.wnmrc.nl/UK

Present Address:

T. Scheenen

Department of Radiology, Radboud University Medical Centre

Nijmegen, Geert Grooteplein 10, 6525 GA Nijmegen,

The Netherlands

\section{Abbreviations}

A Signal amplitude

$B_{0} \quad$ Main magnetic field

$D \quad$ Diffusion coefficient

$\Delta \quad$ Time between two magnetic field gradient pulses in a PFG experiment

$\delta \quad$ Duration of a magnetic field gradient pulse in a PFG experiment

$G \quad$ Amplitude of a magnetic field gradient

$\gamma \quad$ Gyromagnetic ratio $\left(\gamma / 2 \pi\right.$ is $2.68 \times 10^{8} \mathrm{~Hz} / \mathrm{T}$ for $\left.{ }^{1} \mathrm{H}\right)$

$g_{\text {s }} \quad$ Stomatal conductance

FOV Field of view

FT Fourier transform

$H \quad$ Proportionality constant related to tonoplast water permeability

$m \quad$ Turbo factor in a TSE experiment

MRI Magnetic resonance imaging

MSE Multiple spin echo

$N \quad$ Number of pixels in the image matrix

$n_{\text {echo }} \quad$ Echo number in a spin echo train

$N_{\text {echo }}$ Total number of echoes in a spin echo train

NMR Nuclear magnetic resonance

PAM Pulse amplitude modulation chlorophyll fluorometer

PFG Pulsed field gradient

$r \quad$ Radius of a rf detector coil

SE Spin echo

$S / N \quad$ Signal to noise ratio

STE Stimulated echo

$S / V \quad$ Surface to volume ratio of a cell compartment or vessel

$T_{1} \quad$ Spin-lattice or longitudinal relaxation time

$T_{2} \quad$ Spin-spin or transversal relaxation time

$T_{\text {acq }} \quad$ Signal acquisition time

$T E \quad$ Echo time

$T R$ Time between repeated acquisitions 
TSE Turbo spin echo

$v \quad$ Precession frequency

$V \quad$ Pixel volume

\section{Introduction}

The availability of water is one of the major factors that affects plant production, yield, and reproductive success. Water is needed to allow transpiration, $\mathrm{CO}_{2}$ uptake, photosynthesis, and growth. For example, in herbaceous plants the water content is around $95 \%$ and most of the mechanical strength is provided by cells that are rigid only because they are filled with water. Water is passively transported inside plant xylem conduits (vessels and tracheids) in the continuum between soil and atmosphere along a water potential gradient, generated by evaporation. The hydraulic conductivity of the root, stem, and leaves, together with the plants' stomatal regulation, defines the water potential gradients that exist between leaf and root. When this gradient becomes too steep it causes damage either by dehydration of living cells or by cavitation due to tensions (negative pressures) in the water columns of the xylem being too high (Sperry et al. 2002; Mencuccini 2003). Mechanisms are needed to maintain this gradient within a non-damaging range. The most important mechanism is the regulation of the stomatal aperture or stomatal conductance, $g_{\mathrm{s}}$, in the leaves, by increasing the resistance for water vapor leaving the leaves into the atmosphere with lower water content. Changes in $g_{\mathrm{s}}$ will directly affect the uptake of $\mathrm{CO}_{2}$, needed for photosynthesis. There is clear evidence that photosynthesis activity or $\mathrm{CO}_{2}$ assimilation by chloroplasts or leaves is tightly linked to $g_{\mathrm{s}}$, leaf and plant water content, and hydraulic conductance (e.g., Sellers et al. 1997; Hubbard et al. 2001; Tardieu 2003; Buckley 2005).

Even mild water deficits, when relative water content remains above $70 \%$, primarily cause limitation to carbon dioxide uptake because of stomatal closure. With greater water deficits, direct inhibition of photosynthesis occurs (Gupta and Berkowitz 1988; Smirnoff 1993).

Phloem is responsible for the transport of photosynthates such as sucrose from leaves to the rest of the plant. If unloading is inhibited photosynthesis will be decreased. Therefore, there is a strong interrelationship between photosynthesis activity/ $\mathrm{CO}_{2}$ assimilation, plant water status, and xylem and phloem transport/hydraulic conductance (Daudet et al. 2002).

Although these principles are now well known, the dynamics of the interrelationship and integration between these processes on plant level is still lacking. What we need is to be able to measure in intact plants phloem and xylem flow in relation to water content in the surrounding tissues (the storage pools), under normal and under water limiting or even stress conditions (e.g., drought or as a function of phloem loading/unloading mechanisms due to e.g., anoxia), in relation to photosynthesis activity. MRI methods and dedicated hardware have been presented to measure xylem and phloem water transport in relation to water content in different storage tissues (bark, cambial zone, xylem, and parenchyma) non-invasively in the stem of intact plants (Van As 2007; Van As and Windt 2008). In addition, portable NMR (non-spatially resolved) is becoming available for water content measurements in leaves (Capitani et al. 2009). These NMR and MRI methods can be combined with measurements of photosynthesis activity, e.g., monitoring by PAM techniques. In this review, we introduce these NMR and MRI methods and discuss them in relation to spatial and temporal resolution and (sub)cellular water content.

\section{Imaging principles and partial volume effects}

In a homogeneous main magnetic field $B_{0}$, equal spins (e.g., protons of the water molecules) have identical Larmor precession frequency, and a single resonance line in the frequency spectrum is observed at

$v_{0}=(\gamma / 2 \pi) B_{0}$

$\gamma$ is the gyromagnetic ratio that is a characteristic property for each type of spin bearing nuclei. For mobile (liquid) molecules the resonance line is Lorenzian shaped with a width at half maximum inversely proportional to the $T_{2}$, the spin-spin or transverse relaxation time. When a constant magnetic field gradient $G$ is applied superimposed on $B_{0}$, the local magnetic field will become a function of position and identical spins at different positions along this gradient will show different resonance frequencies, each frequency being characteristic for a particular position $r$ :

$v(r)=(\gamma / 2 \pi)\left(B_{0}+G \cdot \mathbf{r}\right)$

For the generation of full two- or three-dimensional NMR images the spin bearing nuclei after excitation have to be labeled for their respective positions by the use of magnetic field gradients in three orthogonal directions, resulting in position dependent frequencies (see e.g., internet book by Hornak 1996-2008). Position labeling by magnetic field gradients can be performed in a variety of ways (see e.g., Callaghan 1993). Depending on the actual sequence used, the position labeling process will take some time. In the frequently used, so-called 2D Fourier Transform (FT) spinecho (SE) sequence, acquisition of the signal occurs at a certain time $T E$ (echo-time) after the excitation of the spin system (Fig. 1). During that time the signal will decay according to the $T_{2}$ relaxation process: 


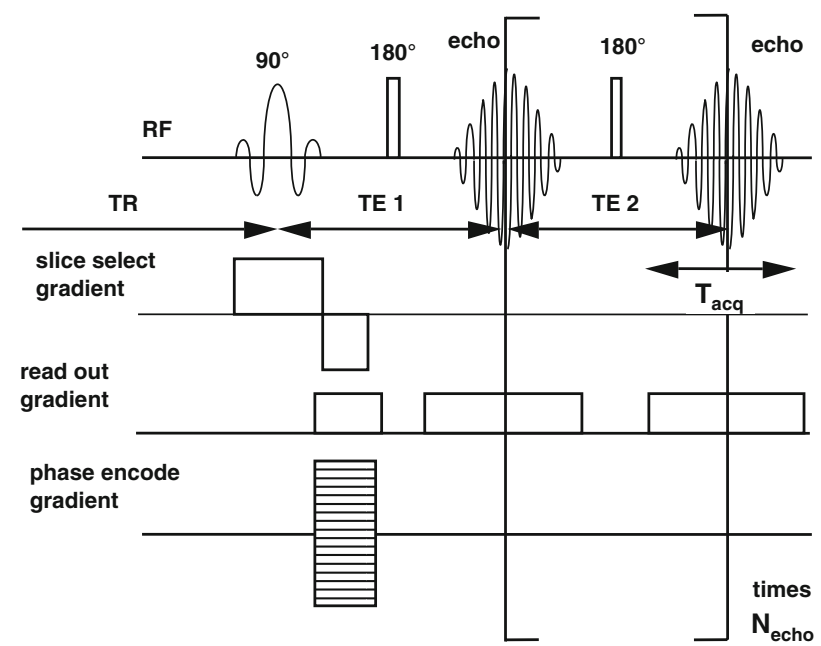

Fig. 1 Scheme of a pulse sequence for multiple spin-echo (MSE) imaging. The echo times TE1 and TE2 may be different in size. The echoes can be acquired separately to obtain images with different $T_{2}$ weighting and can be used to calculate local $T_{2}$ values, or the echoes can be added to obtain a higher signal to noise for the images. To obtain a $\mathrm{N} \mathrm{N}$ image matrix, $\mathrm{N}$ data points have to be sampled during the acquisition of each echo. The sequence has to be repeated for $\mathrm{N}$ different values of the phase encoding gradient, ranging from $-G_{\max }$ to $G_{\max }$

$$
A(T E)=A_{\text {eff }} \exp \left(-T E / T_{2}\right)
$$

Here $A_{\text {eff }}$ is the signal amplitude directly after excitation. In order to obtain a full two-dimensional image of $N \times N$ pixels, the sequence has to be repeated $N$ times. Therefore, the total acquisition time is $N \times T R$, where $T R$ is the time between each repeat. If $T R$ is long enough, the spin system has restored equilibrium along the magnetic field direction. This process is characterized by the spinlattice or longitudinal relaxation time $T_{1}$. If $T R<3 T_{1}$, the effective signal amplitude, $A_{\text {eff }}$, does not uniquely represent the spin density in each pixel, but depends on a combination of the spin density and $T_{1}$ :

$A_{\text {eff }}=A_{0} \exp \left(-T R / T_{1}\right)$

$A_{0}$ is a direct measure of the amount of spins under observation. As a result, NMR SE image intensity usually depends on a combination of these parameters, reflecting spin density, $T_{1}, T_{2}$, and diffusion behavior, characterized by the diffusion coefficient $D$. Diffusion comes into play due to susceptibility artifacts (distortions of the local magnetic field, e.g., due to small air spaces) and the readout gradient used for position labeling (Edzes et al. 1998).

The spatial resolution is defined by the dimension of the image (the field-of-view, FOV) divided by the number of pixels $N$ (for more details see "Spatial and temporal resolution" section). In common practice, the dimension of a single pixel in an image is larger than a single cell and most pixels will contain information that originates from different sub-cellular compartments or even cells from different tissues, vessels, and tracheids, etc. This is called the partial volume problem. Therefore, the information presented mostly is called "apparent": e.g., apparent $T_{2}, T_{2}$, app, or apparent $D, D_{\text {app. }}$. A number of approaches are discussed to (partly) overcome this problem.

\section{Water content and discrimination of tissues}

In order to measure real water content in the different tissues, we need single parameter maps of $A_{0}$ and info to discriminate between the tissues. Many pulse sequences exist by means of which quantitative maps are obtained that represent single NMR parameters like $A_{0}, T_{2}$, etc. In Multiple Spin-Echo (MSE) MRI (Edzes et al. 1998) a spinecho series is created by applying a train of $180^{\circ} \mathrm{rf}$ pulses that recall or refocus the signal, resulting in a series of echoes (Fig. 1). Each echo is acquired in the presence of a read-out or frequency encoding gradient (cf. Eq. 2) and the whole series of echoes is prepared with a single phase encoding gradient for spatial encoding in the direction of that gradient. By repeating the experiment as a function of different values of the phase encoding gradient a series of spin-echo images is obtained. Single parameter maps can now be processed from the MSE-experiment by assuming a mono-exponential relaxation decay of the signal intensity as a function of $n_{\text {echo }} T E$ in each picture element, pixel:

$A\left(n_{\text {echo }} T E\right)=A_{\text {eff }} \exp \left(-n_{\text {echo }} T E / T_{2, \text { app }}\right)$

$n_{\text {echo }}$ is the echo number, up to the maximum $N_{\text {echo }}$. If $T R>3 T_{1}$ and $T E<T_{2}, A_{\text {eff }}$ equals $A_{0}$ and is a direct measure of the water content times tissue density in a pixel. The resulting single parameter maps are: signal amplitude $\left(A_{0}\right)$ and $T_{2}$ app. An example of an amplitude and $T_{2}$ map, demonstrating the high contrast in $T_{2}$ to resolve different

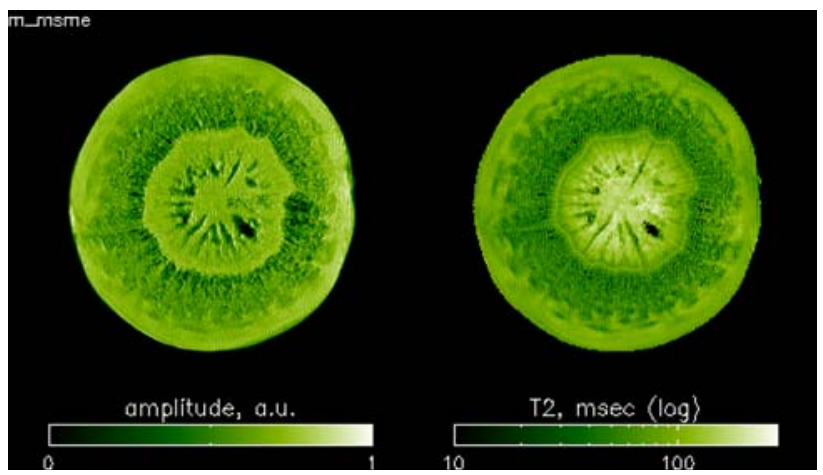

Fig. 2 Amplitude and $T_{2}$ map as a result of a MSE experiment on a carrot tap root on a $3 \mathrm{~T}(128 \mathrm{MHz})$ MRI system. FOV $40 \times 40 \mathrm{~mm}$, $256 \times 256$ image matrix, slice thickness $2 \mathrm{~mm}$ : pixel dimension $156 \times 156 \times 2,000 \mu^{3}$ 
tissue types, are presented in Fig. 2. $T_{2}$-values in big vacuolated plant cells can be found to approach the value of pure water ( $>1.5 \mathrm{~s}$ ) (Edzes et al. 1998). With such long $T_{2}$-values, many spin echoes can be recorded in a single scan (up to 1,000 in a cherry tomato (Edzes et al. 1998)) increasing the total signal-to-noise ratio, $S / N$.

In order to obtain the $A_{0}$ and $T_{2}$ maps, one commonly fits the signal decay in a single pixel by a mono-exponential decay curve. This is in general not correct, due to the partial volume effects. The consequences for water content maps are discussed below.

In general, multi-exponential decay curves are observed for water relaxation measurements in (vacuolated) plant material by non-spatially resolved NMR measurements of homogeneous plant tissue. The different relaxation times can be assigned more or less uniquely to either water in the vacuole (longest $T_{1}$ and $T_{2}$ ), cytoplasm $\left(T_{1}>T_{2}\right.$, both shorter than vacuolar $T_{1}$ and $T_{2}$ ), and cell wall/extra-cellular space ( $T_{2}$ depends strongly on the water content in this compartment, and ranges from about one $\mathrm{ms}$ and higher) (Snaar and Van As 1992; Van Dusschoten et al. 1995; Van der Weerd et al. 2001, 2002). Diffusive exchange within compartments and exchange between compartments, passing membranes, affect the observed relaxation times (Van As 2007; Van As and Windt 2008).

The observed $T_{2}$ (and $T_{1}$ ) of vacuolar water has been demonstrated to depend on the bulk $T_{2}$ in the vacuole $\left(T_{2}\right.$, bulk), and the surface-to-volume ratio, $S / V$, of the vacuole (van der Weerd et al. 2001):

$1 / T_{2, \text { obs }}=(H \times S / V)+1 / T_{2, \text { bulk }}$

The proportionality constant $H$ is directly related to the actual tonoplast membrane permeability for water (van der Weerd et al. 2002; Van As 2007). Equation 6 holds also for water in (xylem) vessels, where $H$ now represents the loss of magnetization at the vessel wall (Homan et al. 2007), demonstrating that $T_{2}$ of vessel water is directly related to vessel radius.

As long as the observed relaxation times are longer than $T E$, the $A_{0}$ maps represent the water density of all water in a pixel and the different tissue types can be discriminated on the basis of their respective $T_{2}$ values (cf. Fig. 2). This condition is easily met for vacuolated plant tissue, where most of the water is in the vacuole, which has relatively long $T_{2}$ values, depending on the size (Eq. 6) and represents most of the water in such cells (Donker et al. 1997; Van der Weerd et al. 2000). It is advisable to use as short as possible $T E$ values to cover the shortest $T_{2}$ values. Most probably extra-cellular water and water in fibers, with short $T_{2}$ values, are hard to observe in MSE type images. In order to obtain $A_{0}$ maps of water with real short $T_{2}$ values, alternative image sequences can be used (Van As et al. 2009; Van Duynhoven et al. 2009).

\section{Xylem and phloem flow}

An example that clearly illustrates how MRI can be used to obtain information from structures that are smaller than a pixel is MRI flow imaging (for some overviews, see MacFall and Van As 1996; Köckenberger 2001; Van As 2007; Van As and Windt 2008). In general, spatial resolution will not be high enough to resolve individual phloem or xylem vessels. As a consequence, pixels that contain flowing water will also contain a significant amount of stationary water. When vessels are very small, as is the case in phloem tissue, the relative amount of flowing water per pixel can be as small as a few percent. The greatest challenge in measuring phloem water transport, therefore, is to distinguish displacement of a small amount of very slowly moving water from a (very) large amount of stationary water showing displacements due to random movement as a result of Brownian motion.

In order to accurately quantify (long distance) xylem and phloem transport, one needs to determine both the direction of the flow, and the actual flow profile, from which the flow velocity, the flux and the flow conducting area can be obtained. At the same time diffusion and flow have to be discriminated. These goals can best be obtained by the use of pulsed magnetic field gradient (PFG) techniques (for some background, see Van As and Windt 2008). In this experiment, a sequence of two magnetic field gradient pulses of duration $\delta$ and equal magnitude $G$ but opposite sign (or equal sign but separated by an $180 \mathrm{rf}$ pulse) label the protons as a function of their position. If the spins remain at exactly the same position the effect of the gradient pulses compensate each other. However, as soon as translation (displacement) motion occurs, the gradients do not exactly compensate each other anymore, resulting in attenuation of the signal amplitude. The amount of this attenuation is determined by the length and amplitude of the gradient pulses, and by the mean translation distance traveled during the interval $\Delta$ between the two gradient pulses.

In order to be able to discern flowing water from randomly diffusing water, $\Delta$ is typically varied from $15 \mathrm{~ms}$ for fast flowing xylem water, to $200 \mathrm{~ms}$ for slow moving phloem water (Scheenen et al. 2001). Linear displacement can be measured by stepping $G$ of the pulsed field gradients $-G_{\max }$ to $+G_{\max }$, as described previously by Scheenen et al. (2000a). After Fourier transformation of the signal as a function of $G$, the complete distribution of displacements (i.e., flow profile) within $\Delta$ in the direction of the gradient is obtained for every pixel of an image. Such a displacement distribution is called a propagator.

Making use of the fact that non-flowing (only diffusion) water results in a propagator that is symmetrical around zero, the signal in the non-flow direction can be mirrored 
around the displacement axis and subtracted from the signal in the flow direction to produce the flow profile of the flowing as well as the stationary water. The resulting flow profiles can then be used to calculate per pixel or in any selected area in an image: the flow conducting area, the average velocity of the flowing water, and by taking the integral of the propagator of the flowing water, the volume flow (cf Fig. 3).

For a reliable determination of the displacement distribution, the NMR signal (echo envelope) has to be measured as a function of at least 32 different $G$ values. It is clear that this takes time. A two-dimensional SE image consisting of $N \times N$ pixels requires $N$ acquisitions to be repeated for phase encoding. Combining this with displacement measurements with 32 gradient steps result in $32 \times N$ acquisitions. If $T R$ is $2 \mathrm{~s}$ and $N=128$, a scan time of at least $136 \mathrm{~min}$ is needed. In order to reduce the acquisition time, displacement imaging has been combined with fast imaging techniques. For turbo-SE this results in a $1 / m$ reduction in scan time as compared to a standard $N \times N$ SE image sequence (Scheenen et al. 2000a). Here $m$ is the turbo factor, equal to the number of spin echoes that can be used for phase encoding in a single scan. It is clear that the number of pixels, $N$, directly determines both spatial and temporal resolution, but acquisition times are in the order of 15-30 min.

The propagator flow imaging approach was used to visualize and quantify xylem flow in tomato (Scheenen et al. 2000a), in stem pieces of chrysanthemum (Scheenen et al. 2000b) and large cucumber plants (Scheenen et al. 2002). While in the last study the authors were able to visualize phloem sap movement, they were not yet able to quantify phloem flow in the same manner as was demonstrated for xylem flow. Windt et al. (2006) further optimized this method as well as the hardware. In this way the dynamics in phloem and xylem flow and flow conducting area were studied in large and fully developed plants: a poplar tree, tomato, tobacco, and castor bean plants. The observed differences for day and night in flow conducting area, which directly relate to xylem and phloem hydraulic conductance, are one of the most striking observations. The phloem fluxes and flow conducting areas showed large differences that roughly corresponded with plant size. The differences in phloem flow velocities between the four species were remarkably small $(0.25-0.40 \mathrm{~mm} / \mathrm{s})$ (Windt et al. 2006).

Plant responses as a function of changes in environmental conditions can now be studied. The method was used by Peuke et al. (2006) to study the effects of cold treatment on mass flow in the phloem. A first example of the effect of an extended dark period (trying to stop photosynthesis and phloem loading) on phloem and xylem flow in Ricinus has been reported (Van As and Windt 2008).
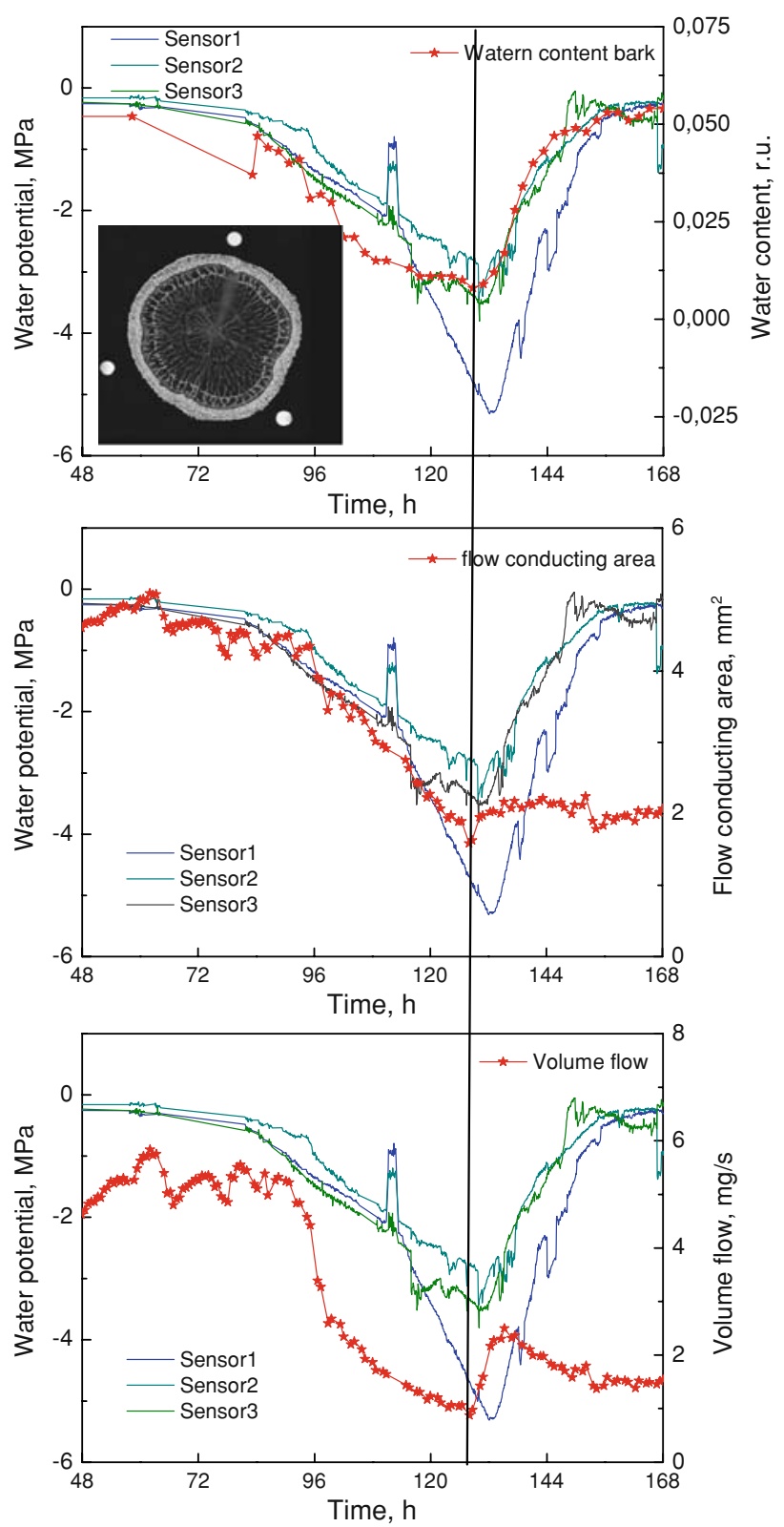

Fig. 3 Example of combined water content (MSE) in one of the storage pools and flow measurements (PFG-TSE) in the stem of a 4 years old oak during a developing drought period, followed by rewatering (indicated by the line). Water content of the bark as (represented by the relative amplitude, the fraction of signal intensity with respect to that of pure water, averaged over all pixels in the mask of the bark as highlighted in the inserted image of the stem), the flow conducting area and volume flow in the active xylem (the xylem ring just inside the bark and the cambial zone). For reference three psychrometer sensor readings of the water potential at three different positions on the stem of the same oak are given (Unpublished results of Homan N, Sperry JS and Van As H)

The method has been applied to study the xylem and phloem flow (and changes therein) in the stalk of a tomato truss during a 8-weeks period of fruit development, revealing that most of the water import to the fruits was through xylem (Windt et al. 2009). Xylem air embolism 
induction and refilling were studied in cucumber (Scheenen et al. 2007), and the effect of root anoxia (trying to limit phloem unloading). Recently, the combination of xylem flow characteristics (flow conducting area, volume flow, and velocity) and changes in water content of the storage pools xylem, cambial zone, and bark during a developing drought stress and after watering in diffuse and ring porous trees (laurel, oak) has been realized (N Homan, JS Sperry, E Gerkema, H Van As, manuscript in prep). An example for oak is given in Fig. 3.

\section{Spatial and temporal resolution}

As stated above spatial resolution depends on the discrimination of the unique frequencies for each position. The differences in frequencies are only dependent on the magnetic field gradient $(\Delta v=\gamma \times G \times \Delta r)$, and not on the main frequency of the spins in the homogeneous magnetic field. In order to be sure that each frequency interval $\Delta v$ contains unique position information, $\Delta v$ must be bigger or at least equal to the line width at half maximum of the resonance line in the homogeneous magnetic field without field gradient, which is dictated by $1 / T_{2} *$. Plant tissue can include intercellular air spaces, resulting in susceptibility artifacts manifest as local magnetic field gradients, $\left.<g_{\mathrm{z}}^{2}\right\rangle$, which shortens the effective $T_{2}$ :

$1 / T_{2} *=1 / T_{2}+\mathrm{f}\left(<g_{\mathrm{z}}^{2}>\right)$

These artifacts increase with increasing field strength: $\left\langle g_{\mathrm{z}}^{2}\right\rangle \sim B_{0}^{2}$. Shorter $T_{2} *$ values increase the necessary $\Delta v$ for a fixed value of $\Delta r$. Applying a strong enough magnetic field gradient $G$ can regulate $\Delta v$. Doing so, there seems to be no limit on spatial resolution. However, an increase in $\Delta v$ results in a decrease of the signal-to-noise ratio $(S / N)$, since the signal per $\Delta r$ is proportional to the number of spins at that position interval, which is fixed. As a result, the signal per $\Delta r$ is smeared out over a larger frequency range $\Delta v$ at increasing $G$, resulting in a decrease in $S / N$.

The $S / N$ is defined by the magnetic field strength, $B_{0}$, the radius of the rf measuring coil (detector), $r$, and details of the experiment, including the measurement time (Homan et al. 2007):

$S / N \sim(V / r) \times B_{0}^{7 / 4} \times\left(N_{\text {av }} \times N_{\text {echo }} / \Delta f\right)^{1 / 2}$

Here $V$ is the pixel volume, and is defined by the number of pixels $N$ within the Field-of-View (FOV), the dimension (in e.g., $\mathrm{cm}$ ) of the image. $N_{\mathrm{av}}$ is the number of averages, $N_{\text {echo }}$ the number of echoes used to construct or calculate the image. $\Delta f$ is the spectral width, representing the frequency range over the given $F O V$. It is inversely related to the dwell time, the time between successive sampled data points. The dwell time times $N$ is the time needed to detect the signal, $T_{\text {acq }}$, and determines the minimal echo time TE. $\Delta f$ divided by the FOV defines $G$. $T_{\text {acq }}$ on its turn is inversely proportional to $G$ during acquisition. The product of $G$ and $T_{\text {acq }}$ defines $\Delta r$.

A number of different approaches can be followed to increase the spatial resolution (minimal $V$ ) at a certain $S / N$, at the same time trying to avoid increasing the measurement time. The $S / N$ of a pixel in an NMR image depends on the amount of water in that pixel. This is the product of tissue water content, tissue density, and pixel volume: the larger the pixel, the lower the spatial resolution of the image, and the higher the $S / N$ of the pixel. In plant stems the thickness of the imaged slice, representing a crosssection of the stem, can be set to a much larger value than the in-plane resolution of the image, because of a large tissue symmetry along the plant stem direction.

Gain can easily be obtained by optimizing $r$ with respect to (part of) the object to be measured. The smaller the $r$, the smaller the pixel volume, and the best approach is to construct rf detector coils that closely fit the object (Scheenen et al. 2002; Windt et al. 2006). Real microscopy, therefore, is limited to small objects. However, small parts on even tall plants can be selected for MRI by the use of dedicated small rf coils, which can easily be build. In this way, e.g., anthers and seed pods, still attached on intact plants, can be imaged with high spatial resolution. An illustration of low field microscopy by the use of optimized hardware (small $r$ ) is presented in Fig. 4. At increasing object size $r$ has to increase and at the same time $N$ has to be increased if one would like to fix $V$. This will result in an increase of measurement time and a decrease in $S / N$.

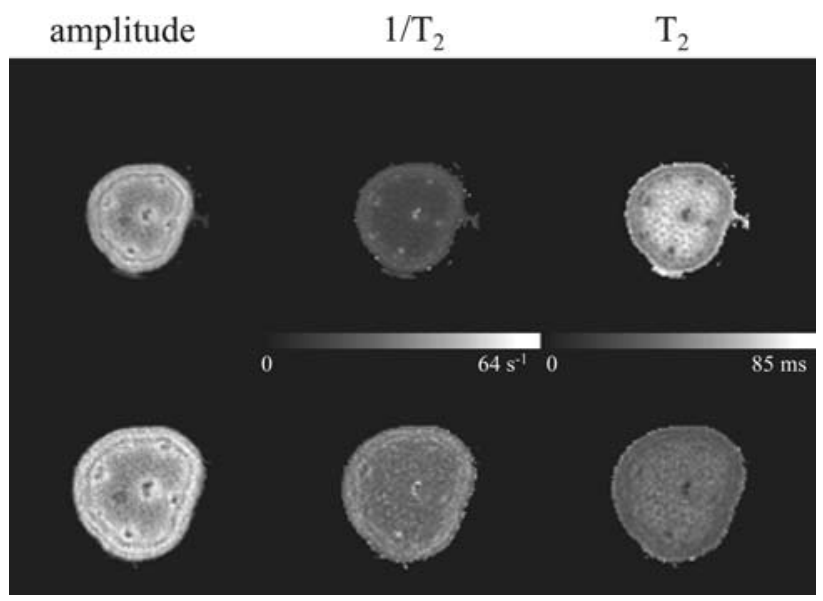

Fig. 4 Amplitude, $1 / T_{2}$ and $T_{2}$ micro-images of leave petiole of geranium measured with a small dedicated rf coil (i.d. $3 \mathrm{~mm}$ ) at $0.7 \mathrm{~T}$ $(30 \mathrm{MHz})$. Parameters: $\Delta f 25 \mathrm{kHz}, \mathrm{TE} 6.6 \mathrm{~ms}, 128 \times 128$ matrix, FOV 5 (first row) en $4 \mathrm{~mm}$ (second row) (resolution $39 \times 39 \times 2500$ and $31 \times 31 \times 2,500 \mu^{3}$, respectively), $\mathrm{N}_{\mathrm{av}} 6$, TR $2.5 \mathrm{~s}, 32 \mathrm{~min}$ total acquisition time 
Next, one can use high $B_{0}$ values. However, for plant tissues with extra-cellular air spaces this results in increased susceptibility artifacts. These artifacts can be overcome by increasing $\Delta f$ (and thus maximum $G$ ), which results in a decrease in $S / N$. At higher $B_{0}$, the effective $T_{2}$ can be (much) shorter than at lower field strength (Donker et al. 1996), limiting the number of measurable echoes $\left(N_{\text {echo }}\right)$, again resulting in lower $S / N$.

Signal averaging over a number of scans also increases the $S / N$, but immediately lengthens the total measurement time and thus reduces the temporal resolution strongly. It is clear that $N$, directly determines both spatial and temporal resolution. In flow imaging a reduced image matrix (e.g. $64 \times 64$ pixels) can be used to reduce temporal resolution, without losing essential flow information.

\section{Do we always need high spatial resolution?}

Resolution, relaxation, and quantification

Since, both a high spatial resolution and a high $S / N$ per pixel are desirable, preferably within an acceptable measurement time, every experiment is a compromise between spatial resolution, $S / N$ and measurement time. The main consideration in this compromise should be the question what information needs to be extracted from the experiment. This information needs to be acquired as accurate as possible within the available measurement time, which is the reason why a high spatial resolution is not always needed. In quantitative $T_{2}$ and proton density imaging and flow imaging, information can be retrieved from several parameters for every pixel, providing a kind of sub-pixel resolution (Norris 2001; Scheenen et al. 2002).

Quantitative $T_{2}$ imaging can even be severely hampered by a high spatial resolution. Movement of protons by selfdiffusion in the time between the large read-out imaging gradients, needed for a high resolution, can attenuate the NMR signal (Edzes et al. 1998). Then, the NMR signal decays not only because of spin-spin relaxation, but also because of diffusion in combination with the imaging gradients. Generally, an exponential decay curve is fitted to the NMR signal decay of every pixel to acquire the $T_{2}$ and the initial signal amplitude at the moment of excitation, reflecting the proton density ( $\approx$ water density). The additional signal attenuation because of diffusion shortens the signal decay time, whereas the initial signal amplitude will remain largely unaffected. In Fig. 4, the difference in $T_{2}$ contrast between two experiments of a geranium petiole (Pelargonium citrosum) with different spatial resolution is shown. At a resolution of $39 \times 39 \times 2,500 \mu^{3} T_{2}$-values of large parenchyma cells in the central cylinder clearly differ from $T_{2}$-values in the cortex, and also the vascular bundles are visible. At a higher resolution of $31 \times 31 \times 2,500 \mu^{3}$ all $T_{2}$-values have decreased due to shortening by diffusion effects, and almost all contrast is gone. The water density images are hardly affected by the additional signal attenuation.

At lower resolution, the $S / N$ of one pixel can be sufficiently high for a meaningful multi-exponential fit (i.e., with acceptable standard deviations of the fitted parameters). This results in two or more water fractions and corresponding relaxation times, which can be assigned to water in sub-cellular compartments within one pixel, creating sub-pixel resolution. In the stem of an intact cucumber plant, a relatively high spatial resolution has been used to distinguish different tissues on the basis of water density and $T_{2}$ of a mono-exponential fit, after which the signal decay curves of a single tissue type were averaged to increase the $S / N$ (Scheenen et al. 2002). The averaged decay curves were fitted to a two-exponential function of which the two water fractions were ascribed to vacuolar water on one hand and water in the cytoplasm and extracellular water on the other hand. Transient changes in $T_{2}$-values of the fractions in the tissues relate to exchange of water over the membranes separating the fractions (the water permeability of the vacuolar and plasmalemma membrane) (van der Weerd et al. 2001).

Combined $T_{1}-T_{2}$ or $D-T_{2}$ measurements, which relate more than one parameter to every pixel of an image, can be used to further improve the sub-pixel information (van Dusschoten et al. 1996; Windt et al. 2007). Recently, an efficient and stable two-dimensional fitting procedure based on a Fast Laplace Inversion algorithm has been introduced (Venkataramanan et al. 2002; Hürlimann et al. 2002), resulting in a two-dimensional correlation plot between $T_{1}$ and $T_{2}$ or $D$ and $T_{2}$, greatly enhancing the discrimination of different water pools (sub-cellular fractions) within a pixel at even relatively low $S / N$. No a priori knowledge about the number or distribution of fractions is necessary.

Not only in quantitative $T_{2}$ imaging, but also in flow imaging experiments, high resolution is not always necessary. The acquisition of propagators enables discrimination between stationary and flowing water at pixel level (see above) (Scheenen et al. 2000b). Even if one or more xylem vessels are captured within one pixel, the signal of the flowing water can still be separated from stationary water. Further improvement for sub-pixel information can be obtained by combined flow- $T_{2}$ measurements (Windt et al. 2007). Then, another compromise has to be made between spatial resolution and the number of gradient steps encoding for flow. The choice depends on the question, what information is more important whether an exact localization of flow or an accurate flow profile? 
Xylem vessels in cucumber plant stems can have diameters up to $350 \mu \mathrm{m}$ (Scheenen et al. 2007), which can be localized much easier than xylem vessels in, e.g., a Chrysanthemum stem with diameters up to $50 \mu \mathrm{m}$ (Nijsse et al. 2001). For large vessels, the amount of flowing water in a pixel is often also large, corresponding to a large integral of the flowing fraction in a pixel-propagator. In this case quantification of the propagators is accurate. With smaller vessels and a distribution of vessel diameters, the amount of flowing water within a pixel is small, resulting in less accurate flow quantification.

\section{Portable NMR and leaf water content}

For understanding water transport and transpiration, leaf hydraulic conductance is crucial. Almost all of the water flux to and within the leaf is lost by transpiration. Therefore, measurements of this flux will allow leaf transpiration to be mapped at either the plant or leaf level. To the best of our knowledge, to date no NMR or MRI flow measurements in leaves have been reported. However, the image of a leaf petiole in Fig. 4 indicates that flow measurements toward a single leaf becomes into reach.

Leaf water content and distribution of leaf water within cell compartments can be approached in a simpler way. In leaves, like all other tissues, multi-exponential $T_{2}$ analyses may yield valuable information with regard to leaf water status and water compartments. Non-imaging NMR has been shown to be able to measure changes in chloroplast water content, in combination with measurements of photosynthesis activity (McCain 1995). Chloroplast volume regulation is a process by means of which chloroplasts import or export osmolytes to maintain a constant volume within a certain range of leaf water potential. Photosynthesis activity rates are directly coupled to changes in chloroplast volumes (Gupta and Berkowitz 1988; Santakumari and Berkowitz 1991). Such studies are especially of interest for plant performance studies under stress conditions in combination with flow imaging and imaging of water content in the storage tissues.

Very recently, a portable unilateral NMR device has been applied to study water content in leaves of intact plants (Capitani et al. 2009). Here, $T_{2}$ measurements at very short $T E$ have been used to overcome the effect on diffusion shortening of the $T_{2}$ due to the very strong background gradient in the unilateral magnet. Extending such measurements by two-dimensional correlation plots between $T_{1}-T_{2}$ or $D-T_{2}$ will greatly enhance the ability to discriminate different pools of water in sub-cellular compartments and reveals the time scale of exchange of water between the different compartments. This approach is very promising to study chloroplast volume regulation in plants under different (water limiting) conditions in relation to photosynthesis monitoring by PAM techniques.

\section{Outlook}

Although, MRI does not deliver a very high spatial resolution, it certainly delivers an abundant amount of information in addition to a reasonable spatial and temporal resolution. Part of this information is very difficult to measure or cannot be measured using other techniques. By the use of dedicated hardware as reported elsewhere (Homan et al. 2007; Van As 2007: Van As and Windt 2008), the xylem and phloem flow and its mutual interaction can be studied.

In addition to water, distribution and flow of nutrients such as sugars are key information to study plant performance. High field NMR and MRI for metabolite mapping and metabolite transport have been demonstrated (Köckenberger et al. 2004; Szimtenings et al. 2003). The combination of water and sugar balance and transport by MRI or NMR non-invasively in the intact plant situation will be the next step to realize.

Relatively cheap imaging set ups based on permanent magnet systems are now becoming available (Haishi et al. 2001; Rokitta et al. 2000). This will greatly stimulate the use of MRI for plant studies.

For NMR flow measurements to be applicable in situ (field situations) quantitative non-spatially resolved (nonimaging) measurements with specifically designed magnets have to be developed. Recently, great improvements in light-weight, portable magnet systems, and spectrometers have been made (Goodson 2006). This trend started with mobile single-sided equipment (Blümich et al. 2008), where a small magnet is placed on the surface of an arbitrarily large object and measures the NMR signal from a small spot close to the surface. This technique is very useful in plant research to study leaf water status (Capitani et al. 2009). A hinged magnet system has been presented, which opens and closes without noteworthy force and is therefore called the NMR-CUFF (Blümler 2007). This versatile instrument weighs only $4 \mathrm{~kg}$ and can for instance be clamped around a tree, branch, or stem of a plant. Mobile equipment like the NMR-CUFF allows studies of plants or plant parts which cannot be investigated in vivo by stationary MRI scanners either because the plants are too big or have to be studied in the field.

Open Access This article is distributed under the terms of the Creative Commons Attribution Noncommercial License which permits any noncommercial use, distribution, and reproduction in any medium, provided the original author(s) and source are credited. 


\section{References}

Blümich B, Perlo J, Casanova F (2008) Mobile single sided NMR. Prog Nucl Magn Reson Spectr 52:197-269; and references therein

Blümler P (2007) The NMR-Cuff: force free, hinged magnet arrangements for portable MRI and EPR. In: Proceedings of 9th international conference on magnetic resonance microscopy, Aachen, Germany

Buckley TN (2005) The control of stomata by water balance. New Phytol 168:275-292

Callaghan PT (1993) Principles of nuclear magnetic resonance microscopy. Clarendon Press, Oxford

Capitani D, Brilli F, Mannina L, Proietti N, Loreto F (2009) In situ investigation of leaf water status by portable unilateral nuclear magnetic resonance. Plant Physiol 149:1638-1647

Daudet FA, Lacointe A, Gaudillère JP, Cruiziat P (2002) Generalized Münch coupling between sugar and water fluxes for modeling carbon allocation as affected by water status. J Theor Biol 214:481-498

Donker HCW, Van As H, Edzes HT, Jans AWH (1996) NMR imaging of white button mushroom (Agaricus bisporus) at various magnetic fields. Magn Reson Imaging 14:1205-1215

Donker HCW, Van As H, Snijder HJ, Edzes HT (1997) Quantitative 1H-NMR imaging of water in white button mushrooms (Agaricus bisporus). Magn Reson Imaging 15:113-121

Edzes HT, van Dusschoten D, Van As H (1998) Quantitative $T_{2}$ imaging of plant tissues by means of multi-echo MRI microscopy. Magn Reson Imaging 16:185-196

Goodson B (2006) Mobilizing magnetic resonance. Phys World 5:28-33

Gupta S, Berkowitz GA (1988) Chloroplast osmotic adjustment and water stress effects on photosynthesis. Plant Physiol 88:200-206

Haishi T, Uematsu T, Matsuda Y, Kose K (2001) Development of a 1.0 T MR microscope using a Nd-Fe-B permanent magnet. Magn Reson Imaging 19:875-880

Homan N, Windt CW, Vergeldt FJ, Gerkema E, Van As H (2007) 0.7 and 3 T MRI and sap flow in intact trees: xylem and phloem in action. Appl Magn Reson 32:157-170

Hornak JP (1996-2008) The basics of MRI. http://www.cis.rit. edu/htbooks/mri/

Hubbard RM, Ryan MG, Stiller V, Sperry JS (2001) Stomatal conductance and photosynthesis vary linearly with plant hydraulic conductance in ponderosa pine. Plant Cell Environ 24:113-121

Hürlimann MD, Venkataramanan L, Flaum C (2002) The diffusionspin relaxation time distribution as an experimental probe to characterize fluid mixtures in porous media. J Chem Phys 117:10223-10232

Köckenberger W (2001) Functional imaging of plants by magnetic resonance experiments. Trends Plant Sci 6:286-292

Köckenberger W, De Panfilis C, Santoro D, Dahiya P, Rawsthoine S (2004) High resolution NMR microscopy of plants and fungi. J Microsc 214:182-189

MacFall JJ, Van As H (1996) Magnetic resonance imaging of plants. In: Shachar-Hill Y, Pfeffer PE (eds) Nuclear magnetic resonance in plant biology, pp 33-76

McCain D (1995) Nuclear magnetic resonance study of spin relaxation and magnetic field gradients in maple leaves. Biophys J 69:1111-1116

Mencuccini M (2003) The ecological significance of long distance water transport: short-term regulation and long-term acclimation across plant growth forms. Plant Cell Environ 26:163-182

Nijsse J, van der Heijden GWAM, van Ieperen W, Keijzer CJ, van Meeteren U (2001) Xylem hydraulic conductivity related to conduit dimensions along chrysanthemum stems. J Exp Bot $52: 319-327$
Norris DG (2001) The effects of microscopic tissue parameters on the diffusion weighted magnetic resonance imaging experiment. NMR Biomed 14:77-93

Peuke AD, Windt CW, Van As H (2006) Effects of cold-girdling on flows in the transport phloem in Ricinus communis: is mass flow inhibited? Plant Cell Environ 29:15-25

Rokitta M, Rommel E, Zimmermann U, Haase A (2000) Portable nuclear magnetic resonance imaging system. Rev Sci Instrum 71:4257-4262

Santakumari M, Berkowitz GA (1991) Chloroplast volume: cell water potential relationships and acclimation of photosynthesis to leaf water deficits. Photosynth Res 28:9-20

Scheenen TWJ, van Dusschoten D, de Jager PA, Van As H (2000a) Microscopic displacement imaging with pulsed field gradient turbo spin-echo NMR. J Magn Reson 142:207-215

Scheenen TWJ, van Dusschoten D, de Jager PA, Van As H (2000b) Quantification of water transport in plants with NMR imaging. J Exp Bot 51:1751-1759

Scheenen TWJ, Vergeldt FJ, Windt CW, de Jager PA, Van As $\mathrm{H}$ (2001) Microscopic imaging of slow flow and diffusion: a pulsed field gradient stimulated echo sequence combined with turbo spin echo imaging. J Magn Reson 151:94-100

Scheenen TWJ, Heemskerk AM, de Jager PA, Vergeldt FJ, Van As H (2002) Functional imaging of plants: a Nuclear magnetic resonance study of a cucumber plant. Biophys J 82:481-492

Scheenen TWJ, Vergeldt FJ, Van As H (2007) Intact plant magnetic resonance imaging to study dynamics in long-distance sap flow and flow-conducting surface area. Plant Physiol 144:1157-1165

Sellers PJ, Dickinson RE, Randall DA et al (1997) Modeling the exchanges of energy, water, and carbon between continents and the atmosphere. Science 275:502-509

Smirnoff N (1993) The role of active oxygen in the response of plants to water deficit and desiccation. New Phytol 125:27-58

Snaar JEM, Van As H (1992) Probing water compartment and membrane permeability in plant cells by proton NMR relaxation measurements. Biophys J 63:1654-1658

Sperry JS, Hacke UG, Oren R, Comstock JP (2002) Water deficits and hydraulic limits to leaf water supply. Plant Cell Environ 25: 251-263

Szimtenings M, Olt S, Haase A (2003) Flow encoded NMR spectroscopy for quantification of metabolite flow in intact plants. J Magn Reson 161:70-76

Tardieu F (2003) Virtual plant: modelling as a tool for the genomics of tolerance to water deficit. Trends Plant Sci 8:9-14

Van As H (2007) Intact plant MRI for the study of cell water relations, membrane permeability, cell-to-cell and long distance water transport. J Exp Bot 58:743-756

Van As H, Windt CW (2008) Magnetic resonance imaging of plants: water balance and water transport in relation to photosynthetic activity. In: Aartsma TJ, Matysik J (eds) Biophysical techniques in photosynthesis II. Springer, Berlin, pp 55-75

Van As H, Homan N, Vergeldt FJ, Windt CW (2009) MRI of water transport in the soil-plant-atmosphere continuum. In: Codd $\mathrm{S}$, Seymour JD (eds) Magnetic resonance microscopy. Wiley-VCH, Weinheim, pp 315-330

van der Weerd L, Vergeldt FJ, de Jager PA, Van As H (2000) Evaluation of algorithms for analysis of NMR relaxation decay curves. Magn Reson Imaging 18:1151-1158

van der Weerd L, Claessens MMAE, Ruttink T et al (2001) Quantitative NMR microscopy of osmotic stress responses in maize and pearl millet. J Exp Bot 52:2333-2343

van der Weerd L, Claessens MMAE, Efdé C, Van As H (2002) Nuclear magnetic resonance imaging of membrane permeability changes in plants during osmotic stress. Plant Cell Environ 25:1538-1549 
van Dusschoten D, de Jager PA, Van As H (1995) Extracting diffusion constants from echo-time-dependent PFG NMR data using relaxation-time information. J Magn Reson A 116:22-28

van Dusschoten D, Moonen CT, de Jager PA, Van As H (1996) Unraveling diffusion constants in biological tissue by combining Carr-Purcell-Meiboom-Gill imaging and pulsed field gradient NMR. Magn Reson Med 36:907-913

Van Duynhoven J, Goudappel GW, Weglarz W (2009) Noninvasive assessment of moisture migration in food products by MRI. In: Codd S, Seymour JD et al (eds) Magnetic resonance microscopy. Wiley-VCH, Weinheim, pp 331-351

Venkataramanan L, Song YQ, Hurlimann MD (2002) Solving Fredholm integrals of the first kind with tensor product structure in 2 and 2.5 dimensions. IEEE Trans Signal Process 50: $1017-1026$

Windt CW, Vergeldt FJ, de Jager PA, Van As H (2006) MRI of long distance water transport: a comparison of the phloem and xylem flow characteristics and dynamics in poplar, castor bean, tomato and tobacco. Plant Cell Environ 29:1715-1729

Windt CW, Vergeldt FJ, Van As H (2007) Correlated displacement$\mathrm{T}_{2}$ MRI by means of a pulsed field gradient-multi spin echo method. J Magn Reson 185:203-239

Windt CW, Gerkema E, Van As H (2009) Most water in the tomato truss is imported through the xylem, not the phloem. An NMR flow imaging study. Plant Physiol; accepted 